\title{
Dynamic Team Contests with Complementary Efforts
}

\author{
Maria Arbatskaya and Hideo Konishi
}

April 30, 2021

\begin{abstract}
In this paper, we study dynamic team contests. In the framework of a Tullock contest between two teams generating impacts according to the Cobb-Douglas effort aggregation function, we examine how equilibrium efforts and winning probabilities depend on the timing of the actions. We show that in contrast to synchronous contests, asynchronous contests with publicly observable actions do not result in the same equilibrium outcome as the one-stage contest; they are strategically unbalancing, leading to more lopsided contests. The results have implications about the design of team contests with complementary efforts.
\end{abstract}

Keywords: team contest, group contest, complementarity in efforts, order of moves, commitment

JEL numbers: C72, D23, D74. 


\section{Introduction}

"Timing is most important...If the timing is right, even a small action will produce a huge impact. If the timing is wrong, even if you push hard, only little will happen. How do you arrive at the timing? This is a complex affair."

- Sadhguru

In this paper, we study the effects of timing of the moves by players in team contests. Team contests provide a useful framework for modeling a variety of competitions between firms, political parties, legal teams, academic teams, and sports teams. In a team contest, each player performs a separate task, and the players' efforts are aggregated into a total team impact that, together with other teams' impacts, determines the chances of winning.

We compare different orders of moves by players in team contests. Suppose player 1 on team 1 can commit to an effort and make it known publicly (to his/her team members and to the members of the competing team). Would the player find it beneficial to commit to a higher or lower effort? How does the possibility of such commitment affect all the players' equilibrium efforts and the teams' chances of winning?

To answer these questions, we employ the model developed by a recent paper by $\mathrm{Lu}$ and $\mathrm{Lu}$ (2020) who consider a two-team contest, in which each team of which is composed of two players assigned to different tasks. Players differ in their marginal costs of making effort. The efforts of players on a team are aggregated by the same Cobb-Douglas function to produce an impact that determines the team's chances of winning through Tullock's contest success function. ${ }^{1}$ With this model, Lu and Lu (2020) considered two different scenarios: (i) all players make efforts simultaneously, and (ii) players make efforts in two stages - in the first stage, players who are assigned to the first task choose efforts; then, after observing stage 1 efforts, players assigned to the second task choose their

\footnotetext{
${ }^{1}$ The Cobb-Douglas effort aggregator function well captures the idea of complementarity between the efforts of players on the same team. Arbatskaya and Mialon (2010) provide an axiomatization of the logit form contest success function with the Cobb-Douglas effort aggregator function in a related context of multi-activity contests where each individual player chooses efforts in multiple activities. The Cobb-Douglas type function arises under a homogeneity axiom, where an equiproportionate change in players' efforts in an activity does not affect players' success probabilities.
} 
effort levels. Lu and Lu (2020) showed that players' effort levels and winning probabilities are the same between (i) and (ii), that is, equilibrium outcomes in one-stage and two-stage synchronous contests are the same. Building on their model, we analyze a general two-stage contest with an arbitrary order of player moves. To find the equilibrium, we quantify the impacts of each player's effort on the efforts of other players using a powerful elasticity formula. With this tool, we demonstrate in Proposition 1 how the equivalence result in $\mathrm{Lu}$ and $\mathrm{Lu}$ (2020) holds in synchronous contests. ${ }^{2}$

We find that it is generally the case that a team can benefit from the strategic choice of timing of individual team members' efforts. When one player on a team is allowed to move earlier than all other players, the player on the stronger team (the favorite team) increases their effort, while the player on the weaker team (the underdog team) decreases their effort. This relaxes competition and reduces the efforts of all players relative to the simultaneous-move game. However, no strategic incentive exists in contests that are overall perfectly balanced in terms of marginal costs of effort (Propositions 2 and 3).

We then compare the odds of winning by team 1 across the two-stage and one-stage contests (Proposition 4). The favorite team has its highest chances of winning when it leads in both tasks and the lowest chances of winning in the synchronous contest. The favorite team has more power when it leads than when it follows. The opposite is true for the underdog team. As a result, in an endogenous timing game of executing the tasks, there is no pure strategy equilibrium in our model. ${ }^{3}$

The rest of the paper is organized as follows. After a brief literature review in the next section, in Section 3, we set up the model and provide a useful elasticity formula for analyzing contests such as Lu and Lu's (2020). In Section

\footnotetext{
${ }^{2}$ Although their model is very different from $\mathrm{Lu}$ and Lu's (2020), Fu, Lu, and Pan (2015) showed that the winning probabilities of teams and individual efforts are all independent of the temporal structure of the component battles (i.e., one-shot or sequential). They considered a majoritarian multi-battle team contest in which players from two rival teams form pairwise matches to compete in distinct component battles - each player fights exactly one battle in the whole contest, much like our synchronous contest.

${ }^{3}$ Dixit (1987) and Yilidirim (2005) considered endogenous timing game in contests and found pure strategy equilibria (see Section 2).
} 
4, we show that their equivalence results for one-stage and two-stage contests hold for a class of synchronous contests. In Section 5, we show that this is generally not the case in contests where some actions are asynchronous. When information about prior choices is publicly available, the contests are generally unbalancing. In Section 6, we compare all the contests in terms of equilibrium chances of winning. Section 7 concludes the paper.

\section{Literature Review}

The literature on dynamic contests is extensive. In a pioneering work, Dixit (1987) considered a strategic timing choice game in a contest played by a favorite and underdog, showing that they choose to move sequentially in equilibrium. Extending Dixit's model by allowing for two rounds of effort decisions, Yilidrim (2005) showed that there are multiple subgame-perfect equilibria, while there is no Stackelberg outcome where the underdog leads and the favorite follows. Ludwig (2012) introduced asymmetric information into Dixit's model, analyzing players' timing of moves. In a model with multiple rounds of play, Harris and Vickers (1987) considered an R\&D race, analyzing how an initial lead by a team affects the subsequent race. Klumpp and Polborn (2006) asked the same question in the context of the US presidential primary races using the Tullock contests, and Konrad and Kovenock (2008) analyzed the dynamic race more generally using all-pay auctions. All of these studies found that the race favors the player who gained a lead in the initial stages. In contrast, Klumpp, Konrad, and Solomon (2019) showed a time-invariance result in a majoritarian Colonel Blotto problem of allocating a given amount of resources to a finite number of battlegrounds. Each player decides how much resources to spend in the next battleground after each battle's result has been revealed. They show that irrespective of the results of the previous battles, the optimal strategy is to spend the same amount of resources.

In a recent innovative paper, Hinnosaar (2018) studied $n$-player sequential contests where each player moves once in an arbitrary order and earlier players' 
efforts are observable by later players. In this model, he found that the total effort is the highest under a fully sequential contest. In contrast, we have teams composed of multiple players; in our team contests, the amount of rent dissipation is the highest in the simultaneous-move contest. Contests played by teams that are composed of multiple players are subject to free-riding among team members. Häfner (2012) considered a tug of war race, which may be played by possibly an infinite number of players, and showed that in his model, there is a unique Markov perfect equilibria. Esteban and Ray (2001) was the first paper that analyzed team contests formally. Assuming that team members' efforts are perfectly substitutable, the authors showed the conditions under which the winning probability of a team increases in its size, despite free-riding incentives. Epstein and Meelem (2009) and Nitzan and Ueda (2011) employed CES effort aggregator functions for team efforts to describe effort complementarities within teams and constant elasticity individual effort costs; they identified the conditions for free-riding incentives to be overcome by effort complementarities. However, these papers are not analyzing dynamic intra- nor inter-team strategic interactions, unlike $\mathrm{Lu}$ and $\mathrm{Lu}$ (2020) and our paper.

The main result from $\mathrm{Lu}$ and $\mathrm{Lu}$ (2020) is that the order in which tasks are performed in team contests does not change the equilibrium efforts as long as tasks are chosen synchronously. In contrast, in multi-activity contests among individual players, which in our framework corresponds to teams composed of a single player who chooses efforts in each task, Arbatskaya and Mialon (2012) showed that when some tasks are chosen before others, players would choose them in a way that relaxes competition in the second stage. This cost-saving strategic incentive is not present when tasks are performed by different team members each maximizing their payoff. When each task is managed separately as in $\mathrm{Lu}$ and $\mathrm{Lu}$ (2020), its impact on the costs of team members is ignored. The current paper illustrates the effects on strategic incentives of having multiple players on each team. 


\section{Model}

Consider a contest among two teams, $i=1,2$, each having two members $j=1,2$ responsible for performing task $j$. We will refer to player $j=1,2$ of team $i=1,2$ as player $i j$. Team members independently choose their effort levels $e_{i j} ; i=1,2$ and $j=1,2$. Players' efforts contribute to their team's chances of winning a prize, which is the public good with a value normalized to 1 . Team members' efforts are aggregated using the Cobb-Douglas function

$$
X_{i}=e_{i 1}^{\alpha_{1}} e_{i 2}^{\alpha_{2}}
$$

where $\alpha_{j} \in(0,1)$ is the sensitivity or discriminatory power of effort in task $j$. We assume Cobb-Douglas impact functions have the same weights on each task across teams and that $\alpha_{1}+\alpha_{2} \leq 1$.

The winning probability of team $i$ is described as a Tullock's (logit) contest success function

$$
P_{i}=\frac{X_{i}}{X_{i}+X_{-i}}=\frac{1}{1+\Theta_{i}},
$$

where $\Theta_{i}=\frac{X_{i}}{X_{-i}}$ is the relative power of team $i$. Player $j$ in team $i$ has the cost of effort described by $c_{i j} e_{i j}$, with $c_{i j}>0$. The expected payoff for player $i j$ of team $i$ is

$$
U_{i j}=P_{i}-c_{i j} e_{i j}=\frac{\Theta_{i}}{1+\Theta_{i}}-c_{i j} e_{i j},
$$

and each team $i$ 's member $j$ chooses his/her effort level $e_{i j}$ to maximize $U_{i j}$. We assume that all of the above is common knowledge among all players. The first-order conditions in the one-stage (static) contest can be written as follows:

$$
\frac{\partial U_{i j}}{\partial e_{i j}}=\frac{1}{\left(1+\Theta_{i}\right)^{2}} \frac{\partial \Theta_{i}}{\partial e_{i j}}-c_{i j}=0
$$

Here, we introduce a key concept of our analysis, the balance of power of the teams in the contest:

$$
\Lambda \equiv P_{1} P_{2}=\frac{X_{1} X_{2}}{\left(X_{1}+X_{2}\right)^{2}}=\frac{\Theta_{i}}{\left(1+\Theta_{i}\right)^{2}}
$$

for $i=1,2$. The balance of power $\Lambda$ is low in lopsided contests, and it reaches its maximum of $1 / 4$ in a symmetric contest, where the two teams have an equal 
chance of winning, $P_{1}=P_{2}=1 / 2$. For future reference, let team 1's relative cost advantage be $\bar{\Theta}_{1} \equiv\left(\frac{c_{21}}{c_{11}}\right)^{\alpha_{1}}\left(\frac{c_{22}}{c_{12}}\right)^{\alpha_{2}}>0$.

\subsection{Elasticity Representation of the First-Order Condi- tions}

In our analysis, it is convenient to use first-order conditions in terms of elasticities. Let $E_{\Theta_{i}, e_{i j}}=\frac{d \Theta_{i}}{d e_{i j}} \frac{e_{i j}}{\Theta_{i}}$ be the elasticity of team $i$ 's (relative) power $\Theta_{i}$ with respect to effort $e_{i j}$. The capitalized $E_{\Theta_{i}, e_{i j}}$ signifies the total elasticity, where we are taking the total (not partial) derivative: $E_{\Theta_{i}, e_{i j}}=\frac{d \Theta_{i}}{d e_{i j}} \frac{e_{i j}}{\Theta_{i}}$ : that is, player ij evaluates the effect of her effort choice $e_{i j}$ by taking the reactions by successive movers (followers) into account. Thus, the first-order condition for player $i j$ 's effort in an elasticity form is as follows:

$$
\frac{d U_{i j}}{d e_{i j}}=E_{\Theta_{i}, e_{i j}} \Lambda \frac{1}{e_{i j}}-c_{i j}=0
$$

or

$$
E_{\Theta_{i}, e_{i j}} \Lambda=c_{i j} e_{i j} .
$$

The expenditures (cost of effort) by player $i j$ are equal to the total elasticity of team $i$ 's (relative) power with respect to effort $e_{i j}$ times the balance of power. The expenditures $c_{i j} e_{i j}$ are higher when the contest is more balanced and when the power of team $i$ is more responsive to changes in task $j$.

To analyze players' strategic actions, we investigate $E_{\Theta_{i}, e_{i j}}$ and $\Lambda$ in contests with different orders of moves. Thanks to the Cobb-Douglas specification of the effort aggregator function, the elasticity of team $i$ 's (relative) power $\Theta_{i}$ with respect to effort $e_{i j}$ in partial differentiation is $\varepsilon_{\Theta_{i}, e_{i j}}=\frac{\partial \Theta_{i}}{\partial e_{i j}} \frac{e_{i j}}{\Theta_{i}}=\alpha_{j}$. Notice that for stage 2 efforts, $E_{\Theta_{i}, e_{i j}}=\varepsilon_{\Theta_{i}, e_{i j}}$ always holds, but this equality is generally not true for player $i j$, who moves in stage 1 if the players in stage 2 can observe $e_{i j}$ before they choose their actions. In the following lemma, we list useful elasticity formulas that will be used to simplify the analysis of the strategic responses in the contest.

Lemma 1. Suppose $g$ and $f$ are $C^{1}$ functions and that $c$ is a constant. Then, we have the following: 
1. for $z(x) \equiv x^{c}$, we have $\varepsilon_{z(x), x}=c$

2. for $z(x) \equiv c g(x)$, we have $\varepsilon_{z(x), x}=\varepsilon_{g(x), x}$

3. for $z(x) \equiv f(x) g(x)$, we have $\varepsilon_{z(x), x}=\varepsilon_{f(x), x}+\varepsilon_{g(x), x}$

4. for $z(x) \equiv \frac{f(x)}{g(x)}$, we have $\varepsilon_{z(x), x}=\varepsilon_{f(x), x}-\varepsilon_{g(x), x}$

5. for $z(x) \equiv f(g(x))$, we have $\varepsilon_{z(x), x}=\varepsilon_{f(y), y} \varepsilon_{g(x), x}$

6. for $z(x) \equiv(g(x))^{c}$, we have $\varepsilon_{z(x), x}=c \varepsilon_{g(x), x}$

For example, consider the contest where player 11 moves first by choosing effort $e_{11}$ in stage 1 . Then, after observing $e_{11}$, other players choose their effort levels simultaneously in stage $2: e_{i j}\left(e_{11}\right)$ for $i j \neq 11$.

To find $E_{\Theta_{1}, e_{11}}$, recall that $\Theta_{1}=\frac{X_{1}}{X_{2}}=\left(\frac{e_{11}}{e_{21}}\right)^{\alpha_{1}}\left(\frac{e_{12}}{e_{22}}\right)^{\alpha_{2}}$ and notice that $\Theta_{1}$ is a function of $e_{11}, e_{12}, e_{21}$, and $e_{22}$ with $\varepsilon_{\Theta_{1}, e_{11}}=\alpha_{1}, \varepsilon_{\Theta_{1}, e_{12}}=\alpha_{2}$, $\varepsilon_{\Theta_{1}, e_{21}}=-\alpha_{1}$, and $\varepsilon_{\Theta_{1}, e_{22}}=-\alpha_{2}$. Denoting the elasticity of responses by the followers to $e_{11}$ with $\varepsilon_{e_{12}, e_{11}}, \varepsilon_{e_{21}, e_{11}}$, and $\varepsilon_{e_{22}, e_{11}}$, we find the following:

$$
E_{\Theta_{1}, e_{11}}=\alpha_{1}+\alpha_{2} \varepsilon_{e_{12}, e_{11}}-\alpha_{1} \varepsilon_{e_{21}, e_{11}}-\alpha_{2} \varepsilon_{e_{22}, e_{11}}
$$

The responses of the followers depend on the extent of the change in the balance of power, $\Lambda$, that player 11's effort brings about. Recall that $\Lambda=\Theta_{1}\left(1+\Theta_{1}\right)^{-2}$, so

$$
E_{\Lambda, e_{11}}=\varepsilon_{\Lambda, \Theta_{1}} E_{\Theta_{1}, e_{11}}
$$

where

$$
\varepsilon_{\Lambda, \Theta_{1}}=\frac{1-\Theta_{1}}{1+\Theta_{1}}
$$

and $E_{\Theta_{1}, e_{11}}$ is stated in equation $(8) ; \varepsilon_{\Lambda, \Theta_{1}} \in(-1,1)$ for $\Theta_{1}>0$.

In the rest of the paper, we consider this team contest with different timing of moves. Our elasticity formula proves useful in understanding players' strategic incentives. In the next section, we review the equivalence result found in $\mathrm{Lu}$ and $\mathrm{Lu}(2020)$. 


\section{Equivalence Result ( $\mathrm{Lu}$ and $\mathrm{Lu} 2020$ )}

$\mathrm{Lu}$ and $\mathrm{Lu}$ (2020) compared the simultaneous move contest (all players choose their effort levels simultaneously) and a synchronous task two-stage contest (task 1's efforts are selected by both teams in stage 1, and task 2's efforts are selected by both teams in stage 2). Somewhat surprisingly, they showed that with a Cobb-Douglas aggregator function, the outcomes of the contests are equivalent, even if the teams and players are asymmetric.

\subsection{One-Stage Contest}

Let's start with the simultaneous move game. When all efforts are chosen simultaneously, we have $E_{X_{i}, e_{i j}}=\varepsilon_{X_{i}, e_{i j}}=\alpha_{j}$. Hence, the first-order conditions (7) can be written as

$$
c_{i j} e_{i j}^{*}=\alpha_{j} \Lambda
$$

where $\Lambda \equiv \Theta_{1}\left(1+\Theta_{1}\right)^{-2}$ and $\Theta_{1} \equiv X_{1} / X_{2}$. This implies $\frac{e_{11}^{*}}{e_{21}^{*}}=\frac{c_{21}}{c_{11}}$ and $\frac{e_{12}^{*}}{e_{22}^{*}}=\frac{c_{22}}{c_{12}}$, and

$$
\Theta_{1}^{*}=\bar{\Theta}_{1} \equiv\left(\frac{c_{21}}{c_{11}}\right)^{\alpha_{1}}\left(\frac{c_{22}}{c_{12}}\right)^{\alpha_{2}} .
$$

Then, from the first-order conditions (7), we have the following:

$$
e_{i j}^{*}=\frac{\alpha_{j}}{c_{i j}} \Lambda\left(\Theta_{1}^{*}\right)=\frac{\alpha_{j}}{c_{i j}} \Lambda\left(\bar{\Theta}_{1}\right) .
$$

\subsection{Synchronous-Task Contests}

Consider a two-stage contest in which $e_{11}$ and $e_{21}$ are chosen at stage 1 and observed by all players at stage 2 , which is when the other efforts are chosen. We start the analysis with the second stage. For the optimal stage 2 efforts $e_{12}^{*}\left(e_{11}, e_{21}\right)$ and $e_{22}^{*}\left(e_{11}, e_{21}\right)$, we have $E_{\Theta_{i}, e_{i j}}=\varepsilon_{\Theta_{i}, e_{i j}}=\alpha_{j}$ because no efforts follow second-stage decisions. The first-order conditions for stage 2 efforts $e_{12}$ and $e_{22}$ are $\alpha_{2} \Lambda=c_{12} e_{12}$ and $\alpha_{2} \Lambda=c_{22} e_{22}$. Hence, the ratio of efforts in task 2 is just the reciprocal of the ratio of the their marginal costs: $\frac{e_{12}^{*}}{e_{22}^{*}}=\frac{c_{22}}{c_{12}}$ for any $e_{11}>0$ and $e_{21}>0$. 
The first-order conditions for stage 1 efforts in elasticity formula are written as

$$
\begin{aligned}
c_{i 1} e_{i 1} & =E_{\Theta_{i}, e_{i 1}} \Lambda \\
& =\left(\alpha_{1}+\alpha_{2} \varepsilon_{e_{i 2}, e_{i 1}}-\alpha_{2} \varepsilon_{e_{i^{\prime}}, e_{i 1}}\right) \Lambda \\
& =\left(\alpha_{1}+\alpha_{2} \varepsilon_{\frac{e_{i 2}}{e_{i^{\prime} 2}}, e_{i 1}}\right) \Lambda=\alpha_{1} \Lambda,
\end{aligned}
$$

where $i \neq i^{\prime}$. The last two equations hold by Lemma 1.4 and $\frac{e_{i 2}}{e_{i^{\prime} 2}, e_{i 1}}=0$ (which comes from $\left.\frac{e_{12}^{*}}{e_{22}^{*}}=\frac{c_{22}}{c_{12}}\right)$, respectively. Then, we also have $\frac{e_{11}^{*}}{e_{21}^{*}}=\frac{c_{21}}{c_{11}}$ and

$$
\Theta_{1}^{*}=\left(\frac{e_{11}^{*}}{e_{21}^{*}}\right)^{\alpha_{1}}\left(\frac{e_{12}^{*}}{e_{22}^{*}}\right)^{\alpha_{2}}=\left(\frac{c_{21}}{c_{11}}\right)^{\alpha_{1}}\left(\frac{c_{22}}{c_{12}}\right)^{\alpha_{2}}=\bar{\Theta}_{1} .
$$

This implies that $P_{1}^{*}=\bar{P}_{1}=\frac{\bar{\Theta}_{1}}{1+\bar{\Theta}_{1}}$. Thus, the synchronous two-stage contest is balance neutral, and we can confirm the equivalence result in $\mathrm{Lu}$ and $\mathrm{Lu}$ (2020) by using the elasticity formulas.

Proposition 1 ( $\mathrm{Lu}$ and $\mathrm{Lu} \mathrm{2020).} \mathrm{In} \mathrm{both} \mathrm{one-stage} \mathrm{and} \mathrm{synchronous} \mathrm{two-}$ stage contests with perfect information, the equilibrium power ratio equals team 1 's relative cost advantage $\bar{\Theta}_{1}$, and equilibrium efforts are $e_{i j}^{*}=\frac{\alpha_{j}}{c_{i j}} \Lambda\left(\bar{\Theta}_{1}\right)$ for all $i, j=1,2$. Team 1's equilibrium winning probability is $P_{1}^{*}=\frac{\bar{\Theta}_{1}}{1+\Theta_{1}}$.

From the elasticity arguments we use in the proof, it is clear that the equivalence theorem in $\mathrm{Lu}$ and $\mathrm{Lu}$ (2020) extends to a model with arbitrary finite numbers of synchronous tasks and stages and to the arbitrary temporal structure of the contest with an arbitrary order, in the spirit of $\mathrm{Fu}, \mathrm{Lu}$, and Pan (2015).

\section{Non-Equivalence Results: Two-Stage Asyn- chronous Contests}

In this section, we show that the equivalence result does not usually extend to contests where there is a task not chosen by the two teams at the same time (synchronously). Consider a contest where player 11 leads all other players. This contest will help us understand all other two-stage contests. 
From, $E_{\Theta_{i}, e_{i j}} \Lambda=c_{i j} e_{i j}$ for $i, j=1,2$, where $\Lambda=\Theta_{1}\left(1+\Theta_{1}\right)^{-2}$. In stage 2, after observing $e_{11}$, the rest of the players choose their effort levels simultaneously. For each $e_{11}$, the optimal stage 2 efforts, the elasticity representation of the first-order conditions $(7)$ for $e_{12}^{*}\left(e_{11}\right), e_{21}^{*}\left(e_{11}\right)$, and $e_{22}^{*}\left(e_{11}\right)$ are:

$$
\begin{aligned}
& c_{12} e_{12}=\alpha_{2} \Lambda \\
& c_{21} e_{21}=\alpha_{1} \Lambda \\
& c_{22} e_{22}=\alpha_{2} \Lambda,
\end{aligned}
$$

because $E_{\Theta_{1}, e_{12}}=\varepsilon_{\Theta_{1}, e_{12}}=\alpha_{2}, E_{\Theta_{2}, e_{21}}=\varepsilon_{\Theta_{2}, e_{21}}=\alpha_{1}$, and $E_{\Theta_{2}, e_{22}}=\varepsilon_{\Theta_{2}, e_{22}}=$ $\alpha_{2}$.

Following the discussions after Lemma 1 (8), player 11's effort $e_{11}$ affects team 1's power as follows:

$$
\begin{aligned}
E_{\Theta_{1}, e_{11}} & =\varepsilon_{\Theta_{1}, e_{11}}+\varepsilon_{\Theta_{1}, e_{12}} \varepsilon_{e_{12}, e_{11}}-\varepsilon_{\Theta_{2}, e_{21}} \varepsilon_{e_{21}, e_{11}}-\varepsilon_{\Theta_{2}, e_{22}} \varepsilon_{e_{22}, e_{11}} \\
& =\alpha_{1}+\alpha_{2} \varepsilon_{e_{12}, e_{11}}-\alpha_{1} \varepsilon_{e_{21}, e_{11}}-\alpha_{2} \varepsilon_{e_{22}, e_{11}},
\end{aligned}
$$

so the first-order condition for $e_{11}$ is

$$
c_{11} e_{11}=E_{\Theta_{1}, e_{11}} \Lambda=\left(\alpha_{1}+\alpha_{2} \varepsilon_{e_{12}, e_{11}}-\alpha_{1} \varepsilon_{e_{21}, e_{11}}-\alpha_{2} \varepsilon_{e_{22}, e_{11}}\right) \Lambda
$$

Thanks to the Cobb-Douglas specification, we can find $\varepsilon_{e_{12}, e_{11}}, \varepsilon_{e_{21}, e_{11}}$, and $\varepsilon_{e_{22}, e_{11}}$ from (11) by totally differentiating the identities with respect to $e_{11}$ (writing in terms of elasticities) and using the elasticity property 2 of Lemma 1:

$$
\begin{aligned}
& E_{e_{12}, e_{11}}=\varepsilon_{e_{12}, e_{11}}=E_{\Lambda, e_{11}} \\
& E_{e_{21}, e_{11}}=\varepsilon_{e_{21}, e_{11}}=E_{\Lambda, e_{11}} \\
& E_{e_{22}, e_{11}}=\varepsilon_{e_{22}, e_{11}}=E_{\Lambda, e_{11}} .
\end{aligned}
$$

That is, the elasticity of the impact of an increase in $e_{11}$ on stage 2 efforts is the same as the elasticity of its impact on the balance of power $\Lambda$. This special property is because of the Cobb-Douglas effort aggregator function, and it simplifies the rest of the analysis tremendously. 
Because the elasticity representation of (9) is $E_{\Lambda, e_{11}}=\varepsilon_{\Lambda, \Theta_{1}} E_{\Theta_{1}, e_{11}}=$ $\frac{1-\Theta_{1}}{1+\Theta_{1}} E_{\Theta_{1}, e_{11}}$, we have the following:

$$
\begin{aligned}
E_{\Theta_{1}, e_{11}} & =\alpha_{1}+\alpha_{2} \varepsilon_{e_{12}, e_{11}}-\alpha_{1} \varepsilon_{e_{21}, e_{11}}-\alpha_{2} \varepsilon_{e_{22}, e_{11}} \\
& =\alpha_{1}-\alpha_{1} E_{\Lambda, e_{11}} \\
& =\alpha_{1}-\alpha_{1} \frac{1-\Theta_{1}}{1+\Theta_{1}} E_{\Theta_{1}, e_{11}} .
\end{aligned}
$$

Solving the above equation for $E_{\Theta_{1}, e_{11}}$, we obtain the following:

$$
E_{\Theta_{1}, e_{11}}=\alpha_{1}\left(1+\alpha_{1} \frac{1-\Theta_{1}}{1+\Theta_{1}}\right)^{-1} \text {. }
$$

Thus the first-order condition for effort $e_{11}(7)$ can be written as follows:

$$
c_{11} e_{11}=\alpha_{1}\left(1+\alpha_{1} \frac{1-\Theta_{1}}{1+\Theta_{1}}\right)^{-1} \Lambda .
$$

By the first-order conditions (7), we have the following:

$$
\frac{e_{11} c_{11}}{e_{21} c_{21}}=\frac{E_{\Theta_{1}, e_{11}}}{\alpha_{1}} \quad \text { and } \quad \frac{e_{12} c_{12}}{e_{22} c_{22}}=1 .
$$

Since $\Theta_{1}=\left(\frac{e_{11}}{e_{21}}\right)^{\alpha_{1}}\left(\frac{e_{12}}{e_{22}}\right)^{\alpha_{2}}$, it follows that

$$
\begin{aligned}
\Theta_{1} & =\left(\frac{E_{\Theta_{1}, e_{11}} c_{21}}{\alpha_{1} c_{11}}\right)^{\alpha_{1}}\left(\frac{c_{22}}{c_{12}}\right)^{\alpha_{2}} \\
& =\left(1+\alpha_{1} \frac{1-\Theta_{1}}{1+\Theta_{1}}\right)^{-\alpha_{1}}\left(\frac{c_{21}}{c_{11}}\right)^{\alpha_{1}}\left(\frac{c_{22}}{c_{12}}\right)^{\alpha_{2}} .
\end{aligned}
$$

Thus, we find that the equilibrium power of team $1, \Theta_{1}=\Theta_{1}^{*}$, is the solution of

$$
\Theta_{1}\left(1+\alpha_{1} \frac{1-\Theta_{1}}{1+\Theta_{1}}\right)^{\alpha_{1}}=\bar{\Theta}_{1}
$$

where $\bar{\Theta}_{1} \equiv\left(\frac{c_{21}}{c_{11}}\right)^{\alpha_{1}}\left(\frac{c_{22}}{c_{12}}\right)^{\alpha_{2}}$ is team 1's relative cost advantage.

The following technical lemma allows us to prove that there exists a unique solution $\Theta_{1}^{*}$ to (19) for any costs $c_{i j}>0$ and weights $\alpha_{j} \in(0,1)$ for $i, j=1,2$; and to show that if $\bar{\Theta}_{1}<1$, then $\Theta_{1}^{*}<\bar{\Theta}_{1}<1$; if $\bar{\Theta}_{1}>1$, then $\Theta_{1}^{*}>\bar{\Theta}_{1}>1$.

Lemma 2. Let $f(x, a) \equiv x\left(1+a \frac{1-x}{1+x}\right)^{a}$ with $x>0$ and $a \in[-1,1]$. Then, $\frac{\partial f(x, a)}{\partial x}>0 ; \frac{\partial f(x, a)}{\partial a}>0$ iff $a(1-x)>0$. Equation $f(x)=\bar{x}$ with any $\bar{x}>0$ has 
a unique solution $x^{*}>0$; if $\bar{x}<1$ then $x^{*}<\bar{x}<1$; if $\bar{x}>1$ then $x^{*}>\bar{x}>1$; if $\bar{x}=1$ then $x^{*}=\bar{x}=1$.

Finally, from the first-order conditions, we have $c_{11} e_{11}^{*}=\kappa \alpha_{1} \Lambda, c_{12} e_{12}^{*}=$ $\alpha_{2} \Lambda, c_{21} e_{21}^{*}=\alpha_{1} \Lambda$, and $c_{22} e_{22}^{*}=\alpha_{2} \Lambda$, where $\kappa \equiv\left(1+\alpha_{1} \frac{1-\Theta_{1}}{1+\Theta_{1}}\right)^{-1}$ and $\Theta_{1}=\Theta_{1}^{*}$ is implicitly defined by (19). Hence, we obtain the proposition below.

Proposition 2 summarizes the subgame-perfect equilibrium in the contest where player 11 leads all other players. It also compares the power of team 1 in this contest to the one in a synchronous contest in which team 1's power is its relative cost advantage, $\bar{\Theta}_{1} \equiv\left(\frac{c_{21}}{c_{11}}\right)^{\alpha_{1}}\left(\frac{c_{22}}{c_{12}}\right)^{\alpha_{2}}$. Since team 1's winning probability of this contest is $P_{1}^{*}=\frac{1}{1+\Theta^{*}}$, we have $P_{1}^{*}=\frac{1}{1+\Theta^{*}}<\frac{1}{1+\bar{\Theta}_{1}}=\bar{P}_{1}$ if $\bar{\Theta}_{1}<1$, and $P_{1}^{*}=\frac{1}{1+\Theta^{*}}>\frac{1}{1+\bar{\Theta}_{1}}=\bar{P}_{1}$ if $\bar{\Theta}_{1}>1$. That is, this dynamic contest enhances the teams' power unbalance by a strategic choice of $e_{11}$.

Proposition 2 (player 11 is the leader). For any costs $c_{i j}>0$ and weights $\alpha_{j}>0, i, j=1,2$, there exists a unique subgame-perfect equilibrium: $e_{11}^{*}=$ $\kappa\left(\Theta_{1}^{*}\right) \frac{\alpha_{1}}{c_{11}} \Lambda\left(\Theta_{1}^{*}\right)$ with $\kappa\left(\Theta_{1}\right)=\left(1+\alpha_{1} \frac{1-\Theta_{1}}{1+\Theta_{1}}\right)^{-1}$ and $e_{12}^{*}=\frac{\alpha_{2}}{c_{12}} \Lambda\left(\Theta_{1}^{*}\right), e_{21}^{*}=$ $\frac{\alpha_{1}}{c_{21}} \Lambda\left(\Theta_{1}^{*}\right)$, and $e_{22}^{*}=\frac{\alpha_{2}}{c_{22}} \Lambda\left(\Theta_{1}^{*}\right)$, where $\Lambda=\Lambda\left(\Theta_{1}\right) \equiv \Theta_{1}\left(1+\Theta_{1}\right)^{-2}$ and the equilibrium power of team $1, \Theta_{1}=\Theta_{1}^{*}$, is implicitly defined as the solution of

$$
f\left(\Theta_{1}, \alpha_{1}\right) \equiv \Theta_{1}\left(1+\alpha_{1} \frac{1-\Theta_{1}}{1+\Theta_{1}}\right)^{\alpha_{1}}=\bar{\Theta}_{1}
$$

with $\bar{\Theta}_{1} \equiv\left(\frac{c_{21}}{c_{11}}\right)^{\alpha_{1}}\left(\frac{c_{22}}{c_{12}}\right)^{\alpha_{2}}$. This dynamic contest is unbalancing in the sense that $P_{i}^{*}=\frac{1}{1+\Theta^{*}}>\bar{P}_{i}=\frac{1}{1+\bar{\Theta}_{i}}$ if team 1 is the favorite team and that $P_{i}^{*}=$ $\frac{1}{1+\Theta^{*}}<\bar{P}_{i}=\frac{1}{1+\Theta_{i}}$ if the team is the underdog.

If team 1 is the favorite, it becomes even more powerful by player 11's commitment to a higher effort $e_{11}$ in stage 1 (overcommitment). If team 1 is the underdog, it becomes less powerful because player 11 exerts less effort. Either way, the contest is unbalancing through the strategic choice of stage 1 effort.

The unbalancing occurs for a reason different than in Arbatskaya and Mialon (2012). In multi-activity contests, unbalancing through the choice of stage 1 
efforts is beneficial for a player because it saves the cost of a stage 2 task. Here, we have each task managed separately by a team player, so the costsaving motive is absent. The reason for a commitment here is to exploit the best responses of the competitors, which are negatively sloped for the underdog team (strategic substitutes) and positively sloped for the favorite team (strategic complements).

By Lemma 2, $f\left(\Theta_{1}, \alpha_{1}\right)$ is an increasing function of $\Theta_{1}$, the equilibrium power of team $1, \Theta_{1}^{*}$, is increasing in $\bar{\Theta}_{1}$. It is also increasing in $\alpha_{1}$ whenever team 1 is the favorite $\left(\Theta_{1}>1\right)$. This means that the favorite team achieves a higher power and probability of winning when the task that the favorite commits to is more influential ( $\alpha_{1}$ is higher). The opposite is true regarding the underdog team's commitment. The underdog's team commitment diminishes its power and chances of winning, and this is all the more the case when the task the underdog is committing to is more influential. The extent of the unbalancing depends on the parameter values. It is impossible to strategically unbalance a contest that is perfectly balanced based on the costs of effort $\left(\bar{\Theta}_{1}=1\right)$.

So far, we have examined synchronous two-stage contests and an asynchronous two-stage contest where a team leads in one task. In the next section, we study other cases of two-task, two-stage contests. We also compare the odds of winning by team 1 across the two-stage and one-stage contests.

\section{Comparing Across Two-Stage Contests}

In this section, we examine two-stage contests with two teams and two tasks. Tasks in a two-stage contest are divided into stage 2 and stage 1 . Without a loss of generality, assume $e_{11}$ is selected in stage 1 . Then, there are five contests to analyze:

C0. (a synchronous two-stage contest) efforts in task 1 are selected in stage 1 and efforts in task 2 are selected in stage 2 ;

C1. one player (player 11) leads, and the rest of the players follow in stage 2; 
C2. one player (player 22) follows, and the rest of the players choose efforts in stage 1 ;

C3. team 1 leads in both tasks; and

C4. teams lead in different tasks: team 1 leads in task 1 and team 2 leads in task 2 .

Contests $\mathrm{C} 0$ - $\mathrm{C} 4$ cover all orders of move in two-stage contests because the results for any other two-stage contests can be obtained by relabeling the teams, tasks, or both. (To switch teams, replace $\Theta_{1}$ with $\Theta_{2}=1 / \Theta_{1}$; to switch tasks, switch $\alpha_{1}$ and $\alpha_{2}$.)

For any $\operatorname{costs} c_{i j}>0$ and weights $\alpha_{j}>0$, we can compute and compare the equilibrium efforts $\left(e_{11}^{*}, e_{12}^{*}, e_{21}^{*}, e_{22}^{*}\right)$ in two-stage contests and in the one-stage contest. We can also compare the power of team 1 and the associated balance of power across the contests. Figure 1 shows the stage 1 and stage 2 efforts in contests $\mathrm{C} 0$ - C4. Proposition 3 summarizers the properties of the equilibria. We show that in general, any asynchronous two-stage contest with publicly observable commitments is strategically unbalancing by the choice of stage 1 tasks, when compared with a synchronous contest $\mathrm{C} 0$ (which is equivalent to the one-stage contest).

Proposition 3. For any costs $c_{i j}>0$ and weights $\alpha_{j}>0, i, j=1,2$, there exists a unique subgame-perfect equilibrium in every two-stage contest $C 0-C 4$. The equilibrium power of team $1, \Theta_{1}=\Theta_{1}^{*}$, is defined by $f\left(\Theta_{1}, a\right)=\bar{\Theta}_{1}$, with $f\left(\Theta_{1}, a\right) \equiv \Theta_{1}\left(1+a \frac{1-\Theta_{1}}{1+\Theta_{1}}\right)^{a}$, where a takes the following values for each case: C0: $a=0, C 1: a=\alpha_{1}$, C2: $a=\alpha_{2}$, C3: $a=\alpha_{1}+\alpha_{2}$, and C4: $a=\alpha_{1}-\alpha_{2}$; stage 1 efforts are $e_{i j}^{*}=\kappa\left(\Theta_{1}^{*}\right) \frac{\alpha_{j}}{c_{i j}} \Lambda\left(\Theta_{1}^{*}\right)$, with $\kappa=\kappa\left(\Theta_{1}\right)=\left(1+a \frac{1-\Theta_{1}}{1+\Theta_{1}}\right)^{-1}>$ 1 iff $a\left(1-\Theta_{1}\right)<0$; and stage 2 efforts are $e_{i j}^{*}=\frac{\alpha_{j}}{c_{i j}} \Lambda\left(\Theta_{1}^{*}\right)$, where $\Lambda\left(\Theta_{1}\right) \equiv$ $\Theta_{1}\left(1+\Theta_{1}\right)^{-2}$. The favorite (underdog) team becomes stronger (weaker) in all asynchronous contests compared with the synchronous contest, unless the contest is perfectly balanced based on costs, $\bar{\Theta}_{1} \equiv\left(\frac{c_{21}}{c_{11}}\right)^{\alpha_{1}}\left(\frac{c_{22}}{c_{12}}\right)^{\alpha_{2}}=1$ or in $C_{4}$ with $\alpha_{1}=\alpha_{2}$. That is, if $\bar{\Theta}_{1}<1$, then $\Theta_{1}^{*}<\bar{\Theta}_{1}<1$; if $\bar{\Theta}_{1}>1$, then $\Theta_{1}^{*}>\bar{\Theta}_{1}>1$. 
The derivations of the subgame-perfect equilibria for contests C2 - C4 follow the same steps as for contest $\mathrm{C} 1$, which was analyzed in the previous section and is omitted here. We know that contest $\mathrm{C} 0$ is equivalent in terms of outcomes to the one-stage contest, in which all tasks are chosen simultaneously. However, contests $\mathrm{C} 1$ - $\mathrm{C} 4$ do not show this equivalence result.

There are strategic incentives to change stage 1 tasks, but no such strategic incentives exist for stage 2 tasks. All stage 1 efforts are overcommitted by the same scaling factor $\kappa$, which is the same for all stage 1 tasks but different across contests $\mathrm{C} 1$ - C4; stage 1 efforts are scaled up by a factor $\kappa>1$ if team 1 is the favorite $\left(\bar{\Theta}_{1}>1\right)$. All stage 1 efforts are scaled down by a scaling factor $\kappa<1$ if team 1 is the underdog $\left(\bar{\Theta}_{1}<1\right)$. A perfectly balanced contest remains perfectly balanced: $\Theta_{1}^{*}=1$ if $\bar{\Theta}_{1}=1$, with no changes in the equilibrium levels of stage 1 tasks.

The unique solution to the equilibrium power of team $1, \Theta_{1}^{*}$, is found numerically. However, the equilibrium properties, such as the over/under commitment by stage 1 players, can be obtained without finding the value for $\Theta_{1}^{*}$. Importantly, assuming $\bar{\Theta}_{1} \neq 1$, the two-stage contest is always unbalancing (the weak team becomes weaker, and the strong team becomes stronger in two-stage contests). The only exception is in contest $\mathrm{C} 4$, where players cross-lead with equally influential tasks, that is, when $\alpha_{1}=\alpha_{2}$. In this case, there is no unbalancing, and there is no strategic advantage to team 1.

The following proposition provides some implications about the timing of moves that a team leader would prefer if this leader cared about their team's probability of winning.

Proposition 4. The favorite team has its highest chances of winning when it leads in both tasks and the lowest chances of winning in the synchronous contest. The favorite team has more power when it leads than when it follows (in one task, in both, or in a more influential task). The opposite is true for the underdog team. 
We always assume $\alpha_{1}>0, \alpha_{2}>0$, and $\alpha_{1}+\alpha_{2} \leq 1$. If $\alpha_{1} \geq \alpha_{2}$ and $\alpha_{1} \leq 2 \alpha_{2}$, this means that the first task is no less influential and no more than twice influential than the second task. Given these constraints, we have $\alpha_{1}+\alpha_{2}>\alpha_{1} \geq \alpha_{2} \geq \alpha_{1}-\alpha_{2} \geq 0$. By Lemma 2 , for any $\Theta_{1}>0$ and $a \in[-1,1]$, $f\left(\Theta_{1}, a\right) \equiv \Theta_{1}\left(1+a \frac{1-\Theta_{1}}{1+\Theta_{1}}\right)^{a}$ is increasing in $\Theta_{1}$, and it is increasing in $a$ if and only if $a\left(1-\Theta_{1}\right)>0$. Assuming $\bar{\Theta}_{1}>1$, equation $f\left(\Theta_{1}^{*}, a\right)=\bar{\Theta}_{1}$ implies the following ranking of contests according to team 1's equilibrium power $\Theta_{1}^{*}$ and probability of winning $p_{1}^{*}$ : $\mathrm{C} 3>\mathrm{C} 1 \geq \mathrm{C} 2 \geq \mathrm{C} 4 \geq \mathrm{C} 0$. The ranking is reversed for team 1 if $\bar{\Theta}_{1}<1$.

We also find that the favorite team's equilibrium power $\Theta_{1}^{*}$ is higher when it leads than when its weaker opponent leads. Hence, the favorite team leading in both tasks results in the highest power and probability of winning for the team. The results are different for the underdog team, which suffers less power loss when it follows than when it leads but has the highest chances of winning in a synchronous contest $\mathrm{C}$.

If we endogenize the order of moves by allowing team leaders to choose the timing of the moves for the team at stage 0 , with the objective of increasing the team's chances of winning, then Proposition 4 implies that there is no pure-strategy equilibrium in the commitment game. From the ranking of probabilities, we know that the best outcome for the favorite team (in terms of the probability of winning) is to assign both tasks to the stage that the underdog does not assign its tasks to (C3). However, if the favorite does so, then the underdog also assigns both tasks to stage 1 , which makes the contest $\mathrm{C} 0$. Then, the favorite assigns its tasks to the other stage, and so on. This is a matchingpennies game. Hence, there is no pure strategy equilibrium in this two-person, zero-sum game. Nash equilibria are necessarily in mixed strategies.

\section{Conclusion}

In this paper, we have studied team contests with a Cobb-Douglas effort aggregation function, in which there are two teams, two tasks, and different timing 
of moves with publicly observed commitments. The problem is complex because when a player makes a choice he/she needs to consider the influence of his/her choice on the choices of all of his/her team members and rivals. Luckily, with the identical Cobb-Douglas effort aggregator function across teams, some effects cancel out. For example, the effect on a player's teammate and their direct opponent at the next stage cancel out because they change by the same percentage and the ratio of their efforts stays the same. Hence, when there are only two stages and two tasks, a player who can precommit would only have to consider the effect of his/her choice on his/her direct opponent if the opponent moves later. No change occurs when he/she moves together with his/her direct opponent (synchronous moves); even though the player has the ability to change the effort levels of his/her teammate and his/her direct opponent, these changes do not affect his/her payoff, which depends on the ratio of efforts in other tasks. Therefore, the Cobb-Douglas effort aggregator function allows us to cancel out all synchronous moves in other tasks. That is, it is possible to extend our analysis to accommodate the multiple-task case: it is clear that the equivalence result in $\mathrm{Lu}$ and $\mathrm{Lu}$ (2020) extends to any synchronous temporal structure in multiple task cases, which follows the spirit of $\mathrm{Fu}, \mathrm{Lu}$, and Pan (2015). In contrast, without synchronous moves for the same tasks, it is also clear that the equivalence theorem does not hold in general.

\section{References}

[1] Arbatskaya, M. and Mialon, H.M., 2010. Multi-activity contests. Economic Theory, 43(1), 23-43.

[2] Arbatskaya, M. and Mialon, H.M., 2012. Dynamic multi-activity contests. Scandinavian Journal of Economics, 114(2), 520-538.

[3] Dixit, A.K., 1987. Strategic behavior in contests. American Economic Review, 77(5), 891-898. 
[4] Epstein, G.S. and Mealem, Y., 2009. Group specific public goods, orchestration of interest groups with free riding. Public Choice, 139 (3-4), 357-369.

[5] Esteban, J. and Ray, D., 2001. Collective action and the group size paradox, American Political Science Review, 95, 663-672.

[6] Fu, Q., Lu, J., and Pan, Y., 2015. Team contests with multiple pairwise battles, American Economic Review, 105(7), 2120-40.

[7] Häfner, S., 2017. A tug-of-war team contest. Games and Economic Behavior, 104, 372-391.

[8] Harris, C., and Vickers, J., 1987. Racing with uncertainty. Review of Economic Studies, 54(1), 1-21.

[9] Hinnosaar, T., 2019. Optimal sequential contests. Working Paper.

[10] Lu, J., and Lu, D., 2020. Task arrangement in team competitions, Economics Letters. 193.

[11] Klumpp, T., and Polborn, M., 2006. Primaries and the New Hampshire effect. Journal of Public Economics, 90(6-7), 1073-1114.

[12] Klumpp, T., Konrad K. A., and Solomon, A., 2019. The dynamics of majoritarian Blotto games. Game and Economic Behavior, 117, 402-419.

[13] Konishi, H., Pan, C.Y., and Simeonov, D., 2019. Equilibrium player choices in team contests with multiple pairwise battles. Working Paper.

[14] Konrad, K. A., and Kovenock, D., 2009. Multi-battle contests. Game and Economic Behavior, 66(1), 256-274.

[15] Nitzan, S. and Ueda, K., 2011. Prize sharing in collective contests. European Economic Review, 55, 678-687.

[16] Ludwig, S., 2012. Contests - a comparison of timing and information structures. Public Choice, 153(3-4), 341-355. 
[17] Yildirim, H., 2005. Contests with multiple rounds. Games and Economic Behavior, 51, 213-227.

\section{Appendix A: Proofs}

Proof of Lemma 2. Let $f(x, a) \equiv x\left(1+a \frac{1-x}{1+x}\right)^{a}$. Then, we have

$$
\begin{aligned}
\frac{\partial f(x ; a)}{\partial x} & =\left(1+a \frac{1-x}{1+x}\right)^{a}-a^{2} x\left(1+a \frac{1-x}{1+x}\right)^{a-1} \frac{2}{(x+1)^{2}} \\
& =\left(1+a \frac{1-x}{1+x}\right)^{a-1}\left(1+a \frac{1-x}{1+x}-x \frac{2 a^{2}}{(x+1)^{2}}\right) \\
& =\left(1+a \frac{1-x}{1+x}\right)^{a-1} \frac{1}{(x+1)^{2}}\left\{(x+1+a)^{2}(1-a)+a^{2}(a+1)\right\} \\
& >0 .
\end{aligned}
$$

Moreover, we have $f(0, a)=0, f(1, a)=1$, and $\lim _{x \rightarrow \infty} f(x, a)=\infty\left(\lim _{x \rightarrow \infty} \frac{1-x}{1+x}=\right.$ -1 and $-1 \leq a \leq 1)$. Therefore, by the intermediate value theorem and $\frac{\partial f(x ; a)}{\partial x}>0$, for any $\bar{x}>0$, there exists a unique solution $x^{*}$ to equation $f(x, a)=\bar{x}$, and $x^{*}>0$; if $\bar{x}>1, f(\bar{x}, a)<\bar{x}$, and if $\bar{x}<1, f(\bar{x}, a)>\bar{x}$. Hence, if $\bar{x}<1$ then $x^{*}<\bar{x}<1$; if $\bar{x}>1$, then $x^{*}>\bar{x}>1$; if $\bar{x}=1$ then $x^{*}=\bar{x}=1$.

\section{Proof of Proposition 2.}

See Case C1 in the Proof of Proposition 3.

\section{Proof of Proposition 3.}

\section{C0: synchronous}

The first-order conditions are $E_{\Theta_{i}, e_{i j}} \Lambda=c_{i j} e_{i j}$ for $i, j=1,2$, where $E_{\Theta_{1}, e_{11}}=$ $\alpha_{1}+\alpha_{2} \varepsilon_{e_{12}, e_{11}}-\alpha_{2} \varepsilon_{e_{22}, e_{11}}=\alpha_{1}, E_{\Theta_{1}, e_{12}}=\alpha_{2}, E_{\Theta_{2}, e_{21}}=\alpha_{1}+\alpha_{2} \varepsilon_{e_{22}, e_{21}}-$ $\alpha_{2} \varepsilon_{e_{12}, e_{21}}$, and $E_{\Theta_{2}, e_{22}}=\alpha_{2}$. Hence, $\frac{e_{12}^{*}}{e_{22}^{*}}=\frac{c_{22}}{c_{12}}$ for any $e_{11}>0$ and $e_{21}>0$. It follows that $\varepsilon_{e_{12}, e_{11}}=\varepsilon_{e_{22}, e_{11}}$, so $E_{\Theta_{1}, e_{11}}=\alpha_{1}+\alpha_{2} \varepsilon_{e_{12}, e_{11}}-\alpha_{2} \varepsilon_{e_{22}, e_{11}}=\alpha_{1}$ and $E_{\Theta_{2}, e_{21}}=\alpha_{1}+\alpha_{2} \varepsilon_{e_{22}, e_{21}}-\alpha_{2} \varepsilon_{e_{12}, e_{21}}=\alpha_{1}$. Equilibrium efforts are such that $c_{i j} e_{i j}^{*}=\alpha_{j} \Lambda\left(\Theta_{1}^{*}\right)$ for all $i, j=1,2, \frac{e_{11}^{*}}{e_{21}^{*}}=\frac{c_{21}}{c_{11}}$, and $\Theta_{1}^{*}=\left(\frac{e_{11}^{*}}{e_{21}^{*}}\right)^{\alpha_{1}}\left(\frac{e_{12}^{*}}{e_{22}^{*}}\right)^{\alpha_{2}}=$ $\left(\frac{c_{21}}{c_{11}}\right)^{\alpha_{1}}\left(\frac{c_{22}}{c_{12}}\right)^{\alpha_{2}}=\bar{\Theta}_{1}$.

\section{C1: player 11 leads}


The first-order conditions are $E_{\Theta_{i}, e_{i j}} \Lambda=c_{i j} e_{i j}$ for $i, j=1,2$, where $E_{\Theta_{1}, e_{11}}=$ $\alpha_{1}+\alpha_{2} \varepsilon_{e_{12}, e_{11}}-\alpha_{1} \varepsilon_{e_{21}, e_{11}}-\alpha_{2} \varepsilon_{e_{22}, e_{11}}, E_{\Theta_{1}, e_{12}}=\alpha_{2}, E_{\Theta_{2}, e_{21}}=\alpha_{1}, E_{\Theta_{2}, e_{22}}=\alpha_{2}$. By fully differentiating the first-order condition for stage 2 tasks with respect to stage 1 effort $e_{11}$, we find that $\varepsilon_{e_{12}, e_{11}}=\varepsilon_{e_{21}, e_{11}}=\varepsilon_{e_{22}, e_{11}}=E_{\Lambda, e_{11}}$. Since $E_{\Lambda, e_{11}}=\frac{1-\Theta_{1}}{1+\Theta_{1}} E_{\Theta_{1}, e_{11}}$, we have $E_{\Theta_{1}, e_{11}}=\alpha_{1}-\alpha_{1} \frac{1-\Theta_{1}}{1+\Theta_{1}} E_{\Theta_{1}, e_{11}}$, which can be solved for $E_{\Theta_{1}, e_{11}}=\alpha_{1}\left(1+\alpha_{1} \frac{1-\Theta_{1}}{1+\Theta_{1}}\right)^{-1}$. It follows that $c_{11} e_{11}^{*}=\kappa \alpha_{1} \Lambda$, $c_{12} e_{12}^{*}=\alpha_{2} \Lambda, c_{21} e_{21}^{*}=\alpha_{1} \Lambda, c_{22} e_{22}^{* *}=\alpha_{2} \Lambda$, where $\kappa=\left(1+\alpha_{1} \frac{1-\Theta_{1}}{1+\Theta_{1}}\right)^{-1}$ and $\kappa>1$ if and only if $\Theta_{1}>1$.

From $\Theta_{1}=\left(\frac{e_{11}}{e_{21}}\right)^{\alpha_{1}}\left(\frac{e_{12}}{e_{22}}\right)^{\alpha_{2}}, e_{11} / e_{21}=\frac{c_{21}}{c_{11}}$, and $e_{12} / e_{22}=\kappa$, we find that $\Theta_{1}=\Theta_{1}^{*}$ is implicitly defined by $\Theta_{1}\left(1+\alpha_{1} \frac{1-\Theta_{1}}{1+\Theta_{1}}\right)^{\alpha_{1}}=\bar{\Theta}_{1}$. By Lemma 2 , the unique solution $\Theta_{1}^{*}$ exists and for any $\operatorname{costs} c_{i j}>0$ and weights $\alpha_{j} \in(0,1)$ for $i, j=1,2$; if $\bar{\Theta}_{1}<1$, then $\Theta_{1}^{*}<\bar{\Theta}_{1}<1$; if $\bar{\Theta}_{1}>1$, then $\Theta_{1}^{*}>\bar{\Theta}_{1}>1$.

\section{C2: player 22 follows}

The first-order conditions are $c_{11} e_{11}=E_{\Theta_{1}, e_{11}} \Lambda, c_{12} e_{12}=E_{\Theta_{1}, e_{12}} \Lambda, c_{21} e_{21}=$ $E_{\Theta_{2}, e_{21}} \Lambda$, and $c_{22} e_{22}=E_{\Theta_{2}, e_{22}} \Lambda$, where $E_{\Theta_{1}, e_{11}}=\alpha_{1}-\alpha_{2} \varepsilon_{e_{22}, e_{11}}, E_{\Theta_{1}, e_{12}}=$ $\alpha_{2}-\alpha_{2} \varepsilon_{e_{22}, e_{12}}, E_{\Theta_{2}, e_{21}}=\alpha_{1}+\alpha_{2} \varepsilon_{e_{22}, e_{21}}$, and $E_{\Theta_{2}, e_{22}}=\alpha_{2}$. That is, $c_{11} e_{11}=$ $\left(\alpha_{1}-\alpha_{2} \varepsilon_{e_{22}, e_{11}}\right) \Lambda, c_{12} e_{12}=\left(\alpha_{2}-\alpha_{2} \varepsilon_{e_{22}, e_{12}}\right) \Lambda, c_{21} e_{21}=\left(\alpha_{1}+\alpha_{2} \varepsilon_{e_{22}, e_{21}}\right) \Lambda$, and $c_{22} e_{22}=\alpha_{2} \Lambda$.

From $\alpha_{2} \Lambda=c_{22} e_{22}$, the full elasticity of $e_{22}$ with respect to $e_{11}$ is $E_{e_{22}, e_{11}}=$ $E_{\Lambda, e_{11}}$. Therefore, we find that $\varepsilon_{e_{22}, e_{11}}=E_{e_{22}, e_{11}}=E_{\Lambda, e_{11}}$. Similarly, $\varepsilon_{e_{22}, e_{12}}=$ $E_{e_{22}, e_{12}}=E_{\Lambda, e_{12}}$, and $\varepsilon_{e_{22}, e_{21}}=E_{e_{22}, e_{21}}=E_{\Lambda, e_{21}}$. Then, $E_{\Theta_{1}, e_{11}}=\alpha_{1}-$ $\alpha_{2} E_{\Lambda, e_{11}}, E_{\Theta_{1}, e_{12}}=\alpha_{2}-\alpha_{2} E_{\Lambda, e_{12}}, E_{\Theta_{2}, e_{21}}=\alpha_{1}+\alpha_{2} E_{\Lambda, e_{21}}$, and $E_{\Theta_{2}, e_{22}}=$ $\alpha_{2}$. Since $E_{\Lambda, e_{i j}}=\frac{1-\Theta_{i}}{1+\Theta_{i}} E_{\Theta_{i}, e_{i j}}$, we have $E_{\Lambda, e_{11}}=\frac{1-\Theta_{1}}{1+\Theta_{1}} E_{\Theta_{1}, e_{11}}, E_{\Lambda, e_{12}}=$ $\frac{1-\Theta_{1}}{1+\Theta_{1}} E_{\Theta_{1}, e_{12}}, E_{\Lambda, e_{21}}=\frac{1-\Theta_{2}}{1+\Theta_{2}} E_{\Theta_{2}, e_{21}}, E_{\Lambda, e_{22}}=\frac{1-\Theta_{2}}{1+\Theta_{2}} E_{\Theta_{2}, e_{22}}=\alpha_{2} \frac{1-\Theta_{2}}{1+\Theta_{2}}$. Then, $E_{\Theta_{1}, e_{11}}=\alpha_{1}-\alpha_{2} \frac{1-\Theta_{1}}{1+\Theta_{1}} E_{\Theta_{1}, e_{11}}, E_{\Theta_{1}, e_{12}}=\alpha_{2}-\alpha_{2} \frac{1-\Theta_{1}}{1+\Theta_{1}} E_{\Theta_{1}, e_{12}}, E_{\Theta_{2}, e_{21}}=$ $\alpha_{1}+\alpha_{2} \frac{1-\Theta_{2}}{1+\Theta_{2}} E_{\Theta_{2}, e_{21}}$, and $E_{\Theta_{2}, e_{22}}=\alpha_{2}$. Note that $\frac{1-\Theta_{2}}{1+\Theta_{2}}=-\frac{1-\Theta_{1}}{1+\Theta_{1}}$. Solving these equalities, we find that $E_{\Theta_{1}, e_{11}}=\kappa \alpha_{1}, E_{\Theta_{1}, e_{12}}=\kappa \alpha_{2}$, and $E_{\Theta_{1}, e_{21}}=\kappa \alpha_{1}$, where $\kappa=\left(1+\alpha_{2} \frac{1-\Theta_{1}}{1+\Theta_{1}}\right)^{-1}$.

By Lemma 2, it follows that for any $\operatorname{costs} c_{i j}>0$ and weights $\alpha_{j}>0$, $i, j=1,2$, there exists a unique subgame-perfect equilibrium: $c_{11} e_{11}^{*}=\kappa \alpha_{1} \Lambda$, 
$c_{12} e_{12}^{*}=\kappa \alpha_{2} \Lambda, c_{21} e_{21}^{*}=\kappa \alpha_{1} \Lambda, c_{22} e_{22}^{*}=\alpha_{2} \Lambda$, where $\kappa=\left(1+\alpha_{2} \frac{1-\Theta_{1}}{1+\Theta_{1}}\right)^{-1}$ and $\kappa>1$ if and only if $\Theta_{1}>1$. From $\Theta_{1}=\left(\frac{e_{11}}{e_{21}}\right)^{\alpha_{1}}\left(\frac{e_{12}}{e_{22}}\right)^{\alpha_{2}}, \frac{c_{11} e_{11}^{*}}{c_{21} e_{21}^{*}}=1$ and $\frac{c_{11} e_{12}^{*}}{c_{22} e_{22}^{* *}}=\kappa$, we find that $\Theta_{1}=\Theta_{1}^{*}$ is implicitly defined by $\Theta_{1}\left(1+\alpha_{2} \frac{1-\Theta_{1}}{1+\Theta_{1}}\right)^{\alpha_{2}}=$ $\bar{\Theta}_{1} ; \Theta_{1}^{*}>\bar{\Theta}_{1}>1$ or $\Theta_{1}^{*}<\bar{\Theta}_{1}<1$.

\section{C3: team 1 leads in both}

The FOCs for stage 2 tasks are $c_{21} e_{21}=\alpha_{1} \Lambda$ and $c_{22} e_{22}=\alpha_{2} \Lambda$. We fully differentiate stage 2 FOCs w.r.t. stage 1 efforts and write this in terms of elasticities: $\varepsilon_{e_{21}, e_{11}}=\varepsilon_{e_{22}, e_{11}}=E_{\Lambda, e_{11}}$ and $\varepsilon_{e_{21}, e_{12}}=\varepsilon_{e_{22}, e_{12}}=E_{\Lambda, e_{12}}$. The FOCs for stage 1 tasks are $c_{11} e_{11}=E_{\Theta_{1}, e_{11}} \Lambda$ and $c_{12} e_{12}=E_{\Theta_{1}, e_{12}} \Lambda$. The elasticity of the impact each stage 1 task has on the power of team 1 undertaking it is: $E_{\Theta_{1}, e_{11}}=$ $\alpha_{1}-\alpha_{1} \varepsilon_{e_{21}, e_{11}}-\alpha_{2} \varepsilon_{e_{22}, e_{11}}=\alpha_{1}-\left(\alpha_{1}+\alpha_{2}\right) E_{\Lambda, e_{11}}=\alpha_{1}-\left(\alpha_{1}+\alpha_{2}\right) \frac{1-\Theta_{1}}{1+\Theta_{1}} E_{\Theta_{1}, e_{11}}$. Similarly, $E_{\Theta_{1}, e_{12}}=\alpha_{2}-\alpha_{1} \varepsilon_{e_{21}, e_{12}}-\alpha_{2} \varepsilon_{e_{22}, e_{12}}=\alpha_{2}-\left(\alpha_{1}+\alpha_{2}\right) E_{\Lambda, e_{12}}=\alpha_{2}-$ $\left(\alpha_{1}+\alpha_{2}\right) \frac{1-\Theta_{1}}{1+\Theta_{1}} E_{\Theta_{1}, e_{12}}$. Solving for $E_{\Theta_{1}, e_{11}}$ and $E_{\Theta_{1}, e_{12}}$, we obtain $E_{\Theta_{1}, e_{11}}=$ $\alpha_{1} \kappa$ and $E_{\Theta_{1}, e_{12}}=\alpha_{1} \kappa$, where $\kappa=\left(1+\left(\alpha_{1}+\alpha_{2}\right) \frac{1-\Theta_{1}}{1+\Theta_{1}}\right)^{-1}$.

The equilibrium efforts are $e_{11}^{*}=\kappa \frac{\alpha_{1} \Lambda}{c_{11}}, e_{12}^{*}=\kappa \frac{\alpha_{2} \Lambda}{c_{12}}, e_{21}^{*}=\frac{\alpha_{1} \Lambda}{c_{21}}, e_{22}^{*}=\frac{\alpha_{2} \Lambda}{c_{22}}$. Then, $\Theta_{1}=\left(\frac{e_{11}}{e_{21}}\right)^{\alpha_{1}}\left(\frac{e_{12}}{e_{22}}\right)^{\alpha_{2}}=\bar{\Theta}_{1} \kappa^{\alpha_{1}+\alpha_{2}}$, where $\bar{\Theta}_{1}=\left(\frac{c_{21}}{c_{11}}\right)^{\alpha_{1}}\left(\frac{c_{22}}{c_{12}}\right)^{\alpha_{2}}$, so $\Theta_{1}=\Theta_{1}^{*}$ is a solution to $\Theta_{1}\left(1+\left(\alpha_{1}+\alpha_{2}\right) \frac{1-\Theta_{1}}{1+\Theta_{1}}\right)^{\alpha_{1}+\alpha_{2}}=\bar{\Theta}_{1}$. By Lemma 2, for any $\operatorname{costs} c_{i j}>0$ and weights $\alpha_{j}>0, i, j=1,2$, there exists a unique subgame-perfect equilibrium $\Theta_{1}^{*}$; if $\bar{\Theta}_{1}<1$ then $\Theta_{1}^{*}<\bar{\Theta}_{1}<1$; if $\bar{\Theta}_{1}>1$, then $\Theta_{1}^{*}>\bar{\Theta}_{1}>1$.

\section{C4: cross-lead}

The FOCs are $c_{11} e_{11}=E_{\Theta_{1}, e_{11}} \Lambda, c_{12} e_{12}=E_{\Theta_{1}, e_{12}} \Lambda, c_{21} e_{21}=E_{\Theta_{2}, e_{21}} \Lambda$, and $c_{22} e_{22}=E_{\Theta_{2}, e_{22}} \Lambda$. Given the timing of moves in the contest, $E_{\Theta_{1}, e_{11}}=$ $\alpha_{1}+\alpha_{2} \varepsilon_{e_{12}, e_{11}}-\alpha_{1} \varepsilon_{e_{21}, e_{11}}, E_{\Theta_{1}, e_{12}}=\alpha_{2}, E_{\Theta_{2}, e_{21}}=\alpha_{1}$, and $E_{\Theta_{2}, e_{22}}=\alpha_{2}-$ $\alpha_{2} \varepsilon_{e_{12}, e_{22}}+\alpha_{1} \varepsilon_{e_{21}, e_{22}}$.

From FOCs for stage 2 efforts $e_{21}$ and $e_{21}$, we have the following responsiveness of stage 2 efforts to stage 1 efforts: $\varepsilon_{e_{12}, e_{11}}=\varepsilon_{e_{21}, e_{11}}=E_{\Lambda, e_{11}}=$ $\frac{1-\Theta_{1}}{1+\Theta_{1}} E_{\Theta_{1}, e_{11}}$ and $\varepsilon_{e_{12}, e_{22}}=\varepsilon_{e_{21}, e_{22}}=E_{\Lambda, e_{22}}=\frac{1-\Theta_{2}}{1+\Theta_{2}} E_{\Theta_{2}, e_{22}}$. Then, $E_{\Theta_{1}, e_{11}}=$ $\alpha_{1}+\left(\alpha_{2}-\alpha_{1}\right) \frac{1-\Theta_{1}}{1+\Theta_{1}} E_{\Theta_{1}, e_{11}}$, so $E_{\Theta_{1}, e_{11}}=\kappa \frac{\alpha_{1}}{1+\left(\alpha_{1}-\alpha_{2}\right) \frac{1-\Theta_{1}}{1+\Theta_{1}}}$, where $\kappa \equiv\left(1+\left(\alpha_{1}-\alpha_{2}\right) \frac{1-\Theta_{1}}{1+\Theta_{1}}\right)^{-1}$. 
Similarly, $E_{\Theta_{2}, e_{22}}=\alpha_{2}+\left(\alpha_{1}-\alpha_{2}\right) \frac{1-\Theta_{2}}{1+\Theta_{2}} E_{\Theta_{2}, e_{22}}$, and therefore $E_{\Theta_{2}, e_{22}}=\kappa \alpha_{2}$.

We have $c_{11} e_{11}=\kappa \alpha_{1} \Lambda, c_{12} e_{12}=\alpha_{2} \Lambda, c_{21} e_{21}=\alpha_{1} \Lambda$, and $c_{22} e_{22}=\kappa \alpha_{2} \Lambda$, where $\kappa \equiv\left(1+\left(\alpha_{1}-\alpha_{2}\right) \frac{1-\Theta_{1}}{1+\Theta_{1}}\right)^{-1} ; \Theta_{1}=\left(\frac{e_{11}}{e_{21}}\right)^{\alpha_{1}}\left(\frac{e_{12}}{e_{22}}\right)^{\alpha_{2}}=\bar{\Theta}_{1} \kappa^{\alpha_{1}-\alpha_{2}}$, where $\bar{\Theta}_{1}=\left(\frac{c_{21}}{c_{11}}\right)^{\alpha_{1}}\left(\frac{c_{22}}{c_{12}}\right)^{\alpha_{2}}$, and therefore $\Theta_{1}=\Theta_{1}^{*}$ is a solution to $\Theta_{1}\left(1+\left(\alpha_{1}-\alpha_{2}\right) \frac{1-\Theta_{1}}{1+\Theta_{1}}\right)^{\alpha_{1}-\alpha_{2}}=$ $\bar{\Theta}_{1}$. By Lemma 2 , for any costs $c_{i j}>0$ and weights $\alpha_{j}>0, i, j=1,2$, there exists a unique subgame-perfect equilibrium $\Theta_{1}^{*}$; if $\bar{\Theta}_{1}<1$ then $\Theta_{1}^{*}<\bar{\Theta}_{1}<1$; if $\bar{\Theta}_{1}>1$, then $\Theta_{1}^{*}>\bar{\Theta}_{1}>1 ; \kappa>1$ iff $\left(\alpha_{1}-\alpha_{2}\right)\left(1-\Theta_{1}\right)<0 . \square$

Proof of Proposition 4. By Proposition 3, the equilibrium power of team 1, $\Theta_{1}=\Theta_{1}^{*}$, is defined by $f\left(\Theta_{1}, a\right) \equiv \Theta_{1}\left(1+a \frac{1-\Theta_{1}}{1+\Theta_{1}}\right)^{a}=\bar{\Theta}_{1}$, where $a$ equals 0 , $\alpha_{1}, \alpha_{2}, \alpha_{1}+\alpha_{2}$, and $\alpha_{1}-\alpha_{2}$ for contests C0 - C4, correspondingly. Since we assume $\alpha_{1}>0, \alpha_{2}>0$, and $\alpha_{1}+\alpha_{2} \leq 1$, we have $a \in[-1,1]$. By Lemma 2, for any $\Theta_{1}>0$ and $a \in[-1,1], \frac{\partial f\left(\Theta_{1} ; a\right)}{\partial \Theta_{1}}>0 ; \frac{\partial f\left(\Theta_{1} ; a\right)}{\partial a}>0$ iff $a\left(1-\Theta_{1}\right)>0$. Equation $f\left(\Theta_{1}^{*}, a\right)=\bar{\Theta}_{1}$ then implies that $\frac{\partial \Theta_{1}^{*}}{\partial a}>0$ if $\Theta_{1}>\bar{\Theta}_{1}>1$ and $\frac{\partial \Theta_{1}^{*}}{\partial a}<0$ if $\Theta_{1}<\bar{\Theta}_{1}<1$.

We can then rank the contests $\mathrm{C} 0$ - C4 in terms of $\Theta_{1}^{*}$ for $\bar{\Theta}_{1}>1$. If $\alpha_{1} \geq \alpha_{2}$ and $\alpha_{1} \leq 2 \alpha_{2}$, then $\alpha_{1}+\alpha_{2}>\alpha_{1} \geq \alpha_{2} \geq \alpha_{1}-\alpha_{2} \geq 0$ and $\mathrm{C} 3>\mathrm{C} 1 \geq \mathrm{C} 2 \geq \mathrm{C} 4 \geq \mathrm{C} 0$. If $\alpha_{1}>2 \alpha_{2}$, then $\alpha_{1}+\alpha_{2}>\alpha_{1}>\alpha_{1}-\alpha_{2}>\alpha_{2}>0$ and $\mathrm{C} 3>\mathrm{C} 1>\mathrm{C} 4>\mathrm{C} 2>\mathrm{C} 0$. If $\alpha_{1}<\alpha_{2}$, then $\alpha_{1}+\alpha_{2}>\alpha_{2}>\alpha_{1}>0>\alpha_{1}-\alpha_{2}$ and $\mathrm{C} 3>\mathrm{C} 2>\mathrm{C} 1>\mathrm{C} 4>\mathrm{C} 0$. In any case, the favorite has the highest $\Theta_{1}^{*}$ and $p_{1}^{*}=1 /\left(1+1 / \Theta_{1}^{*}\right)$ in contest $\mathrm{C} 3$ and the lowest $\Theta_{1}^{*}$ and $p_{1}^{*}$ in contest $\mathrm{C} 0$.

The ranking is reversed for $\bar{\Theta}_{1}<1$. If $\alpha_{1} \geq \alpha_{2}$ and $\alpha_{1} \leq 2 \alpha_{2}$, then $\mathrm{C} 3<\mathrm{C} 1 \leq \mathrm{C} 2 \leq \mathrm{C} 4 \leq \mathrm{C} 0$. If $\alpha_{1}>2 \alpha_{2}$, then $\mathrm{C} 3<\mathrm{C} 1<\mathrm{C} 4<\mathrm{C} 2<\mathrm{C} 0$. If $\alpha_{1}<\alpha_{2}$, then $\mathrm{C} 3<\mathrm{C} 2<\mathrm{C} 1<\mathrm{C} 4<\mathrm{C} 0$. In any case, the underdog has the highest $\Theta_{1}^{*}$ and $p_{1}^{*}=1 /\left(1+1 / \Theta_{1}^{*}\right)$ in contest $\mathrm{C} 0$ and the lowest $\Theta_{1}^{*}$ and $p_{1}^{*}$ in contest $\mathrm{C} 3$.

Next, we compare the power of team 1 when it leads and when the other team leads. Recall that $\Theta_{1}^{*}(a)$ is defined by $f\left(\Theta_{1}, a\right) \equiv \Theta_{1}\left(1+a \frac{1-\Theta_{1}}{1+\Theta_{1}}\right)^{a}=\bar{\Theta}_{1}$. For a given $\Theta_{1}>0, f\left(\Theta_{1}, a\right)<f\left(\Theta_{1},-a\right)$ if $a>0$ and $\Theta_{1}>1$. Hence, $\Theta_{1}^{*}(a)>\Theta_{1}^{*}(-a)$ if $a>0$ and $\Theta_{1}>1$. Similarly, $\Theta_{1}^{*}(a)<\Theta_{1}^{*}(-a)$ if $a>0$ and $\Theta_{1}<1$. These results hold when $a=\alpha_{1}>0, a=\alpha_{1}+\alpha_{2}>0$, and $a=\alpha_{1}-\alpha_{2}>0 . \square$ 